\title{
Una evolución de la mirada sobre la complejidad de los objetos matemáticos
}

\author{
Vicenç Font \\ vfont@ub.edu \\ https://orcid.org/0000-0003-1405-0458 \\ Universitat de Barcelona (UB) \\ Barcelona, España. \\ Luis R. Pino-Fan \\ luis.pino@ulagos.c1 \\ https://orcid.org/0000-0003-4060-7408 \\ Universidad de Los Lagos (UdL) \\ Osorno, Chile. \\ Adriana Breda \\ adriana.breda@ub.edu \\ https://orcid.org/0000-0002-7764-0511 \\ Universitat de Barcelona (UB) \\ Barcelona, España
}

Recibido: 28/03/2020 Aceptado: 20/04/2020

\begin{abstract}
Resumen
Se explica la evolución de una agenda de investigación que se inició con el artículo "Objetos, prácticas y ostensivos asociados. El caso de la cisoide”, publicado el 2001, donde se pretendía mostrar la ingenuidad del punto de vista que consideraba a las representaciones ostensivas de los objetos matemáticos simplemente como diferentes significantes de objetos a-históricos y también se ponía énfasis en la importancia que tenía, para la Didáctica de la Matemáticas, problematizar la visión platónica sobre los objetos matemáticos. Para ello, se tomó como contexto de reflexión la evolución de las diferentes representaciones de la cisoide. El objetivo del artículo tenía aspectos en común con los principios asumidos por el Enfoque Ontosemiótico, por esta razón, el primer autor se fue interesando en la agenda de investigación que proponía dicho enfoque, en particular en cómo conceptualizar, desde una perspectiva pragmatista, el papel de las representaciones, y participó activamente en dar una respuesta, que no fuese platónica, a una de las preguntas que ha sido el motor de desarrollo del Enfoque Ontosemiótico: ¿qué es un objeto matemático y cuál es su significado en una determinada institución? La respuesta a esta pregunta iba de la mano con otro aspecto: la importancia para la Didáctica de la Matemáticas de tener en cuenta la complejidad de los objetos matemáticos, lo cual llevó a los tres autores de este trabajo a investigar sobre la complejidad de diferentes objetos matemáticos, así como sobre la comprensión que tienen los estudiantes de dicha complejidad. En este artículo, además de explicar el camino recorrido que se acaba de comentar, se explica cómo se ha incorporado la idea de "tener en cuenta la complejidad del objeto matemático a enseñar" en algunas experiencias de formación de profesores, donde han participado los autores.
\end{abstract}

Palabras clave: Objeto Matemático. Complejidad del Objeto Matemático. Idoneidad Didáctica. Enfoque Ontosemiótico. 


\title{
Uma evolução da visão sobre a complexidade dos objetos matemáticos
}

\begin{abstract}
Resumo
Explica-se a evolução de um processo de pesquisa iniciado com o artigo "Objetos, práticas ostensivas associadas. O caso do cissoide", publicado em 2001, pretendia-se mostrar a ingenuidade do ponto de vista que considerava as representações ostensivas de objetos matemáticos simplesmente como diferentes significantes de objetos a-históricos, além de enfatizar a importância que eles tinham, para a Didática da Matemática, de problematizar a visão platônica de objetos matemáticos. Para isso, tomou-se como contexto de reflexão, a evolução das diferentes representações do cissoide, cujo objetivo apresentava aspectos em comum com os princípios assumidos pela Abordagem Ontossemiótica. Por essa razão, o primeiro autor foi interessando-se no processo de pesquisa que propunha dita abordagem, em particular em como conceituar, a partir de uma perspectiva pragmatista, o papel das representações, e participou ativamente em dar uma resposta, que não fosse platônica, a uma das perguntas que foi o motor de desenvolvimento da Abordagem Ontossemiótica: o que é um objeto matemático e qual o seu significado em uma determinada instituição? A resposta a esta pergunta vai ao encontro de outro aspecto: a importância para a Didática da Matemática de se ter em conta a complexidade dos objetos matemáticos, o qual levou aos três autores de este trabalho a investigar sobre a complexidade de diferentes objetos matemáticos, assim como sobre a compreensão de dita complexidade apresentadas pelos estudantes e professores. Neste artigo, além de mostrar o caminho percorrido que se acaba de comentar, explica-se como se incorporou a ideia de "ter em conta a complexidade do objeto matemático a ensinar" em algumas experiências de formação de professores, onde participaram os três autores.
\end{abstract}

Palavras-chave: Objeto Matemático. Complexidade do Objeto Matemático. Adequação didática. Abordagem Ontosemiótica.

\section{An evolution of the point of view on the complexity of mathematical objects}

\begin{abstract}
This work explains the evolution of a research agenda that began with the article "Objects, practices and associated ostensive. The cissoids case", published in 2001, which was intended to show the ingenuity of the view that regarded the ostensive representations of mathematical objects simply as different signifiers of objects ahistorical and emphasis is also placed on the importance he had, for the Didactics of Mathematics, problematize the Platonic vision of mathematical objects. For this, the evolution of the different representations of the cissoid was taken as a context of reflection. The objective of the article had aspects in common with the principles assumed by the Ontosemiotic Approach, for this reason, the first author became interested in the research agenda that said approach proposed, particularly in how to conceptualize, from a pragmatist perspective, the role of the representations, and actively participated in giving a non-Platonic answer to one of the questions that has been the driving force behind the development of the Ontosemiotic Approach: what is a mathematical object and what is its meaning in a particular institution? The answer to this question is going to meet another aspect: the importance for didactics of mathematics to take into account the complexity of mathematical objects, which led to the three authors of this work to investigate the complexity of different mathematical objects, as well how about the understanding of this complexity presented by students and teachers. In this article, in addition to showing what has just been
\end{abstract}


said, it explains how the idea of "taking into account the complexity of the mathematical object to be taught" was incorporated in some teacher training experiences, where the three authors participated.

Keywords: Mathematical Object. Complexity of the Mathematical Object. Didactic suitability. Ontosemiotic Approach.

\section{Introducción}

En su conferencia plenaria de apertura en el PME-42, en el año de 2018, Mogens Niss caracterizó el artículo de la revista de educación matemática ideal y, en contraste con él, hizo un llamamiento a la comunidad de educación matemática sobre la importancia de publicar, entre otros, documentos no empíricos que se centran en un tema, en comparar o vincular marcos teóricos, o en presentar y analizar métodos (Niss, 2018). En la misma conferencia plenaria, Niss recordó la distinción de Arcavi (2000) entre investigación dirigida por problemas e investigación teórica en educación matemática. Siguiendo la sugerencia de Niss, este artículo es un documento que se aleja del informe de investigación típico (o ideal) ya que estamos informando sobre nosotros mismos, los autores, en particular de la evolución de la mirada sobre la complejidad de los objetos matemáticos del primer autor del artículo y de su impacto en el grupo de investigación que coordina, del cual forman parte los otros dos autores; ya que se trata de una narrativa de una evolución de la mirada sobre la complejidad de los objetos matemáticos, y de cómo ésta ha incidido en las investigaciones de los autores, en especial sobre las que están relacionadas con la formación de profesores. Este tipo de artículo, que abarca un recorrido sobre un amplio período, nos pareció especialmente adecuado para esta edición de la revista Paradigma, que publica un número especial conmemorando sus 40 años de vida.

El punto de partida de la evolución que se narra se inicia con la participación del primer autor de este artículo en la elaboración del artículo Objetos, prácticas y ostensivos asociados. El caso de la cisoide, conjuntamente con Rosa Peraire, publicado el año 2001 en la revista Educación Matemática (Font y Peraire, 2001). El objetivo de dicho artículo era mostrar la ingenuidad del punto de vista que consideraba a las representaciones ostensivas de los objetos matemáticos simplemente como diferentes significantes de objetos a-históricos, puesto que sus repercusiones didácticas tendían a minusvalorar la importancia de las diferentes representaciones ostensivas y las traducciones entre ellas en la producción de sentido. Para conseguir este objetivo, se realizó una investigación que puso el foco en la evolución histórica de las diferentes representaciones ostensivas de los objetos matemáticos y se tomó el caso de la 
cisoide como contexto de reflexión. De este objeto matemático, se estudió la aparición de sus diferentes formas de representación, las traducciones entre ellas, y los diferentes programas de investigación en los que éstas se enmarcaban.

En este artículo, después de esta introducción, hay una segunda sección donde se explica el contexto académico y conceptual en el que se desarrolló la investigación que se describe en el artículo de la cisoide, así como el interés y relevancia del tema en ese entonces. En la siguiente sección se explica la evolución -y adopción de nuevas perspectivas en el marco del Enfoque Ontosemiótico de la Cognición e Instrucción Matemáticos (EOS) (Godino, Batanero y Font, 2007; 2019) - de la problemática iniciada en el artículo sobre la cisoide. En la cuarta sección se explica la relación del contenido del artículo sobre la cisoide con el trabajo actual de los tres autores de este artículo.

La metodología usada tiene aspectos de las siguientes metodologías: 1) narrativa (una mirada a una historia de uno mismo), 2) autoetnografía (una mirada a sí mismo dentro de un contexto más amplio) y autoestudio (una mirada a uno mismo en acción, generalmente dentro de contextos educativos) (Hamilton, Smith \& Worthington, 2008), en las que se privilegia el yo/nosotros.

\section{Contexto en el que se desarrolló la investigación sobre la cisoide. interés y relevancia de la problemática abordada}

Cuando se escribió este artículo sobre la cisoide, los dos autores estábamos interesados, por una parte, en ampliar la investigación sobre las diferentes representaciones de un objeto matemático con análisis históricos y no limitarla, solamente, a investigaciones cognitivas sobre el efecto que producen las diferentes representaciones sobre la comprensión que genera el alumno y, por otra parte, en focalizar la investigación sobre la complejidad asociada a los objetos matemáticos.

En el año 2001 nos interesaba ilustrar, utilizando como contexto de reflexión el caso de la cisoide, la complejidad de las relaciones que se establecen entre un objeto matemático, sus ostensivos asociados, las técnicas que permiten manipular estos ostensivos y las situaciones en las que se usa el objeto (juntamente a sus ostensivos y técnicas asociadas) para organizar fenómenos. 
En el año 2001 nos interesaba generar una línea de investigación, sobre el papel de las diferentes representaciones de los objetos matemáticas en la enseñanza de las matemáticas, de tipo pragmatista que fuese una alternativa a la investigación de tipo cognitivo sobre las representaciones, que era la dominante entonces. En aquella época, las investigaciones en didáctica de las matemáticas de tipo cognitivo entendían la comprensión de un objeto matemático, básicamente, en términos de integración de representaciones mentales, junto con las relaciones funcionales entre ellas. Esta integración es la que aseguraba la competencia en el uso de las representaciones externas asociadas al objeto matemático. Desde esta perspectiva, un objetivo central en la enseñanza de las matemáticas consistía en conseguir que "los estudiantes sean capaces de pasar desde una representación a otra sin caer en contradicciones" (Hitt, 1998, p. 124). Este objetivo era asumido por muchos investigadores en la Didáctica de las Matemáticas y lo podíamos encontrar formulado en términos parecidos, tanto para la enseñanza como para el aprendizaje, en muchas publicaciones. Por ejemplo, con relación al aprendizaje, en Duval (2002, p. 318) se decía: “La conversión de representaciones es un problema crucial en el aprendizaje de las matemáticas". Para las investigaciones de tipo cognitivo la dualidad interno/externo era una noción clave y las representaciones cognitivas internas (o mentales) se introducían como una herramienta teórica para caracterizar las cogniciones complejas que podían construir los estudiantes sobre las representaciones externas. Las representaciones internas no se podían observar directamente, sino que eran inferidas a partir de conductas observables sobre las representaciones externas.

En cambio, los autores del artículo sobre la cisoide estábamos interesado en el desarrollo de una línea de investigación en la Didáctica de las Matemáticas sobre las representaciones de los objetos matemáticos de tipo pragmatista, que también daba mucha importancia al uso de diferentes representaciones, aunque las razones para ello eran diferentes a las que daban las investigaciones de tipo cognitivo. Mientras que en las segundas las representaciones se analizaban básicamente desde la perspectiva representacional (algo por algo), en las primeras primaba el aspecto instrumental (lo que se podía hacer con la representación). El valor representacional lleva a entender la representación de una manera elemental o unitaria "algo" por "algo". En cambio, el valor instrumental lleva a entender la representación de una manera sistémica, como el detonante de un sistema complejo de prácticas que dicha representación permite realizar (otra representación diferente permitiría otro tipo de prácticas). 


\section{Evolución y adopción de nuevas perspectivas en el marco del EOS de la problemática iniciada en el artículo sobre la cisoide}

En el artículo sobre la cisoide del 2001 no había ninguna referencia a investigaciones realizadas usando como marco teórico el Enfoque Ontosemiótico de la Cognición e Instrucción Matemáticos, pero el objetivo del artículo tenía muchos aspectos en común con los principios asumidos por este enfoque. Por esta razón, el primer autor de este artículo se fue interesando en la agenda de investigación que proponía dicho enfoque, en particular en cómo se conceptualizaba el papel de las representaciones en el EOS, lo cual le llevó a colaborar en los siguientes artículos sobre las representaciones desde la perspectiva del EOS: Font, Godino y D’Amore (2007 y 2010) y Godino y Font (2010). En estos artículos se afronta uno de los aspectos clave que plantea el uso de las representaciones: la naturaleza y la diversidad de los objetos que desempeñan el papel de representación y de los objetos representados. En estos artículos se muestra como la noción de función semiótica y la ontología matemática elaborada por el EOS nos permiten dar una respuesta de tipo pragmatista a esta cuestión. En particular, nos permiten afrontar la siguiente problemática: 1) La naturaleza de los objetos que intervienen en las representaciones, 2) La distinción entre representaciones internas y externas. 3) El problema de la representación del elemento genérico. 4) El papel que desempeñan las representaciones de un mismo objeto en su emergencia y 5) Procesos de comprensión y su relación con la traducción entre diferentes representaciones.

Otro aspecto que estaba presente, de manera incipiente, es este artículo sobre la cisoide era la importancia para la Didáctica de la Matemáticas de problematizar la visión platónica sobre los objetos matemáticos. El punto de vista platónico sobre las representaciones ostensivas de los objetos matemáticos considera que éstas son secundarias y relativamente neutras, ya que se consideran como diferentes significantes de objetos matemáticos a-históricos. El efecto que producen las diferentes representaciones ostensivas en la producción de sentido es un tema que no preocupa en demasía a la concepción platónica, ya que este posible efecto corresponde al contexto de descubrimiento y no al contexto de justificación. La problematización del punto de vista platónico iba de la mano con otro aspecto que también estaba presente, de manera incipiente, es este artículo sobre la cisoide: la importancia para la Didáctica de la Matemáticas 
de tener en cuenta la complejidad de los objetos matemáticos, entendida como pluralidad de significados.

El hecho de que el EOS fuese uno de los marcos teóricos que más se había interesado en reflexionar sobre la complejidad de los objetos matemáticos y en buscar una explicación de cómo éstos emergen en el aula que no fuese de tipo platónico, llevó al primer autor del artículo sobre la cisoide a reflexionar sobre estos aspectos utilizando como marco teórico el EOS. En concreto, participó activamente en dar una respuesta a una de las preguntas que ha sido el motor de desarrollo del EOS: ¿Qué es un objeto matemático y cuál es su significado en una determinada institución? (Godino, Batanero y Font, 2019).

\section{La emergencia de los objetos matemáticos en el EOS}

En Font, Godino y Gallardo (2013) se da una respuesta a la pregunta anterior en la que la noción de complejidad del objeto matemático y la de articulación de los componentes de dicha complejidad juegan un papel esencial. Estos autores consideran que el camino por el cual los objetos matemáticos emergen a partir de las prácticas es complejo y deben ser distinguidos, al menos, dos niveles de emergencia. En un primer nivel, emergen representaciones, definiciones, proposiciones, procedimientos, problemas y argumentos (llamados objetos primarios en el EOS), que se organizan en configuraciones llamadas epistémicas. Con relación a la naturaleza de dichos objetos primarios, en el EOS, en consonancia con la filosofía de la matemática de Wittgenstein (1978), se considera que el tipo de existencia de las definiciones, proposiciones y procedimientos es el que tienen las reglas convencionales. Desde este punto de vista, los enunciados matemáticos son reglas (gramaticales) para el uso de cierto tipo de signos porque de hecho se usan como reglas. No describen propiedades de objetos matemáticos con algún tipo de existencia independiente de las personas que quieren conocerlos y del lenguaje que se usa para conocerlos, aunque lo pueda parecer.

Para explicar cómo emergen los objetos primarios se usa la metáfora subir una escalera para resaltar que la práctica matemática se puede considerar como subir una escalera, donde el escalón en el que nos apoyamos para realizar la práctica es una configuración de objetos primarios ya conocida, mientras que el escalón superior al que accedemos, como resultado de la práctica realizada, es una nueva configuración de objetos primarios en la que alguno (o 
algunos) de dichos objetos no era conocido antes. Los nuevos objetos primarios aparecen como resultado de la práctica matemática y se convierten en objetos primarios institucionales gracias, entre otros procesos, a procesos de institucionalización.

Si bien es cierto que en el EOS se adopta un punto de vista convencionalista sobre la naturaleza de los objetos matemáticos, no se ignora que implícitamente se está sugiriendo en los procesos de enseñanza, explícita o implícitamente, una visión descriptiva/realista de las matemáticas. Para poder explicar cómo se genera dicha visión es necesario considerar un segundo nivel de emergencia, en el cual emerge un objeto matemático (por ejemplo, el objeto función), que es considerado como un objeto que se representa por diferentes representaciones, que puede tener varias definiciones equivalentes, que tiene propiedades, etc. Esta segunda emergencia es el resultado de diferentes factores. Los principales son los siguientes:

1) La objetividad de las matemáticas. En las matemáticas escolares se realiza un discurso sobre las matemáticas que, de manera explícita o implícita, envía a los alumnos el mensaje de que las matemáticas es una ciencia cierta, verdadera, objetiva, etc. Es habitual en el discurso escolar explicar la objetividad de las matemáticas, explícita o implícitamente, sugiriendo que, si bien las matemáticas son el resultado de la actividad de resolución de problemas de las diferentes sociedades humanas, su verdad, objetividad, etc., no depende de las personas que las han desarrollado.

2) El éxito predictivo de las ciencias que usan las matemáticas. Este éxito se utiliza, de manera explícita o implícita, como argumento para avalar la existencia de los objetos matemáticos. Con este argumento se está potenciando, sobre todo, el realismo ontológico.

3) Simplicidad, intencionalidad y reificación. En la vida cotidiana es útil suponer que las diferentes experiencias que se tienen de un objeto, por ejemplo, una silla, se deben a que existe un objeto silla que es la causa de dichas experiencias. De la misma manera, resulta muy cómodo considerar que existe un objeto matemático que es representado por diferentes representaciones, que se puede definir por varias definiciones equivalentes, que tiene propiedades, etc.

4) La metáfora del objeto en el discurso del profesor. Tal como se explica en Font, Godino, Planas y Acevedo (2010), en el EOS se considera que la metáfora objeto juega un papel relevante para considerar a los objetos matemáticos como objetos con un cierto tipo de existencia. Se trata de una metáfora conceptual que tiene sus orígenes en nuestras experiencias con los objetos físicos y permite la interpretación de los eventos, actividades, emociones, ideas... 
como si fueran entidades reales con propiedades. La metáfora objeto está siempre presente en el discurso de los profesores, porque en él las entidades matemáticas se presentan como objetos con propiedades. En el discurso matemático es habitual el uso de determinadas expresiones metafóricas de esta metáfora conceptual que sugieren que los objetos matemáticos no son construidos, sino que son objetos preexistentes descubiertos; nos referimos a palabras del tipo describir, hallar, encontrar, buscar, etc.

5) Diferenciación entre ostensivos y no ostensivos. Tal como se argumenta en Font, Godino, Planas y Acevedo (2010), en el discurso matemático, es posible: a) hablar de un ostensivo que representa un no ostensivo que no existe (por ejemplo, podemos decir que $f^{\prime}(a)$ no existe debido a que el gráfico de $f(x)$ tiene una forma puntiaguda en $x=a$ ), y b) la diferenciación del objeto matemático de sus representaciones. Ambos aspectos llevan a los estudiantes a interpretar los objetos matemáticos como diferentes de sus representaciones ostensivas. Este tipo de discurso que producimos dentro de la clase de matemáticas nos lleva a inferir la existencia del objeto como algo independiente de su representación.

Estos cinco factores, entre otros, generan en el aula, implícita o explícitamente, la visión descriptiva-realista de las matemáticas que considera: 1) que las proposiciones matemáticas describen propiedades de objetos matemáticos y 2) que dichos objetos tienen algún tipo de existencia independiente de las personas que los conocen y del lenguaje que se usa para conocerlos. Dicha visión resulta difícil de evitar ya que las razones que llevan a generarla están actuando constantemente y de manera muy sutil. Más que una posición filosófica asumida conscientemente, se trata de una forma de entender los objetos matemáticos que está presente de manera implícita.

Según el EOS, el objeto matemático derivada (por ejemplo) hay que situarlo en el segundo nivel de emergencia de objetos considerado en este enfoque. Se trata de la emergencia de una referencia global asociada a diferentes configuraciones de objetos primarios, las cuales permiten realizar prácticas matemáticas en diferentes contextos -en los cuales, por ejemplo, la derivada se ha interpretado como límite, como pendiente de la recta tangente o como velocidad instantánea, además como un operador que transforma una función en otra-, lo cual lleva a entender que la derivada se puede definir de diversas formas, representar de formas diferentes, etc. El resultado, según el EOS, es que se considera que hay un objeto, llamado derivada, que juega el papel de referencia global de todas las configuraciones de objetos primarios. Ahora 
bien, dicha referencia global, en la actividad matemática, se concreta en una configuración de objetos primarios determinada. Por tanto, lo que se puede hacer con este objeto de segundo nivel está determinado por esta configuración. En el EOS, el objeto que juega el papel de referencia global se puede considerar como único por razones de simplicidad y, a la vez, como múltiple ya que, metafóricamente, se puede decir que estalla en una multiplicidad de objetos primarios agrupados en diversas configuraciones.

La mirada sobre la complejidad de los objetos matemáticos que tiene el EOS permite reformular la visión ingenua de que hay un mismo objeto matemático con diferentes representaciones. Lo que hay es un sistema complejo de prácticas, que permiten resolver problemas, en las cuales el objeto matemático (secundario) no aparece directamente, aquello que sí aparece son representaciones del objeto (secundario), diferentes definiciones, proposiciones y propiedades del objeto, procedimientos y técnicas que se aplican al objeto y argumentos sobre el objeto matemático (configuraciones epistémicas de objetos primarios en términos del EOS). Dicho de otro modo, a lo largo de la historia se han ido generando diferentes configuraciones epistémicas de objetos primarios para el estudio del objeto matemático (secundario), algunas de las cuales han servido para generalizar a las preexistentes.

\section{Relación de la evolución de la mirada sobre la complejidad de los objetos matemáticos con la agenda actual de investigación de los autores}

En esta sección explicamos la relación de la evolución de la mirada sobre la complejidad de los objetos matemáticos con las investigaciones actuales de los autores. En concreto se explican las investigaciones realizadas sobre la complejidad de diferentes objetos matemáticos, en particular la derivada y la antiderivada, así como la comprensión que tienen los estudiantes de dicha complejidad. También se explica cómo se ha incorporado el componente representatividad de la complejidad del objeto matemático a enseñar en el criterio de idoneidad epistémica, uno de los criterios de idoneidad didáctica propuestos por el EOS para valorar un

proceso de instrucción, y algunas experiencias de formación de profesores, donde han participado los autores, en las que se ha enseñado el uso de dicho componente.

\section{Investigaciones sobre la complejidad de diferentes objetos matemáticos}


El primer autor de este artículo participó en diferentes investigaciones para profundizar en la complejidad de diferentes objetos matemáticos: números naturales (Godino, Font, Wilhelmi y Arrieche, 2009), media aritmética (Rondero y Font, 2015), límite (Contreras, García y Font, 2012), Optimización (Balcaza, Contreras y Font, 2017) y colaboró, con la tercera autora, para caracterizar la complejidad del Teorema de Thales (Font, Breda y Seckel, 2017) y, con el segundo, para caracterizar la complejidad de la derivada (Pino-Fan, Castro, Godino y Font, 2013; Pino-Fan, Godino y Font, 2011) y de la antiderivada (Gordillo, Pino-Fan, Font y PonceCampuzano, 2018); así como la comprensión que tienen los estudiantes de dicha complejidad, con el segundo y tercer autor (Pino-Fan, Font, Gordillo, Larios y Breda, 2018; Pino-Fan, Godino y Font, 2018). A su vez, la tercera autora, profundizó en la complejidad de las inecuaciones (Monje, Seckel y Breda, 2018) y el segundo autor en la antiderivada (Gordillo y Pino-Fan, 2016).

Para el objeto matemático derivada, Pino, Godino y Font (2011) caracterizan su complejidad mediante nueve configuraciones de objetos primarios: 1) tangente en la matemática griega; 2) variación en la edad media; 3) métodos algebraicos para hallar tangentes; 4) concepciones cinemáticas para el trazado de tangentes; 5) ideas intuitivas de límite para el cálculo de máximos y mínimos; 6) métodos infinitesimales en el cálculo de tangentes; 7) cálculo de fluxiones; 8) cálculo de diferencias; y 9) derivada como límite. En Pino, Castro, Godino y Font (2013) se utilizan estas nueve configuraciones para la reconstrucción del significado global de la derivada, el cual es utilizado para valorar la representatividad del significado pretendido en el currículo de Bachillerato de México (a partir de las configuraciones de objetos primarios activadas en las prácticas matemáticas propuestas tanto en el Plan de Estudios como en los libros de texto de dicho nivel).

La caracterización de la complejidad de la derivada realizada en Pino-Fan, Godino y Font (2011) facilita tener elementos para diseñar cuestionarios que permiten caracterizar la comprensión de los estudiantes, futuros profesores o profesores en servicio sobre la derivada. En Pino-Fan, Godino y Font (2018), se diseñó un cuestionario para determinar la comprensión de futuros profesores sobre la derivada en el que se incluyeron tareas que activan los diversos significados parciales de la derivada caracterizados en Pino-Fan, Godino y Font (2011).

En Gordillo y Pino-Fan (2016) la complejidad de la antiderivada se caracteriza mediante cuatro configuraciones de objetos primarios relacionadas con cuatro problemas fundamentales: 
a) el problema geométrico de las tangentes de una curva y la cuadratura de la misma; b) el problema de la relación fluxiones - fluentes; c) el problema sobre la relación de los diferenciales y las sumatorias; y d) el problema de la identificación de funciones elementales. La caracterización de dicha complejidad permite tener elementos para diseñar cuestionarios que permiten caracterizar la comprensión de los estudiantes, futuros profesores o profesores en servicio sobre la antiderivada. En Gordillo, Pino-Fan, Font y Ponce (2018) y en Pino-Fan, Font, Gordillo, Larios y Breda (2018), se diseñó un cuestionario para determinar la comprensión de los estudiantes universitarios sobre la antiderivada en el que se incluyeron tareas que activan los diversos significados parciales de la antiderivada caracterizados en Gordillo y Pino (2016).

En estas investigaciones se llegó a la conclusión de que los profesores debían tener en cuenta la complejidad de los objetos matemáticos que enseñaban para conseguir una enseñanza más eficaz, lo cual llevó a los dos autores de este artículo a interesase por la manera de incorporar la problemática de la complejidad de los objetos matemáticos en la formación de profesores:

Nuestra propuesta de reconstrucción del significado global de la derivada resulta especialmente importante puesto que el diseño, implementación y evaluación de planes de formación matemática y de procesos instruccionales sobre un contenido matemático específico, requieren un estudio en profundidad sobre el significado de los objetos matemáticos que componen dicho contenido. Tal estudio debe aportar criterios para seleccionar los problemas y prácticas matemáticas a incluir en los planes y procesos de formación, según las necesidades sociales y profesionales del grupo de personas a quien se dirigen. Es decir, es a partir del significado holístico de un objeto, que se determina cuál o cuáles serán los significados pretendidos, implementados y evaluados, en una práctica educativa específica. De esta manera, es indudable que el significado global de la derivada es pieza clave del conocimiento didácticomatemático del profesor (Pino-Fan, Godino y Font, 2011, p. 175).

En particular nos llevó a desarrollar, juntamente con otros investigadores que también utilizan como referente teórico el EOS, el constructo idoneidad didáctica de un proceso de instrucción-siendo en la revista Paradigma donde se publicó uno de los primeros artículos que desarrollaban dicho constructo (Godino, Bencomo, Font y Wilhelmi, 2006). Dicho constructo se compone en seis criterios de idoneidad parciales, uno de los cuales es el criterio de idoneidad epistémica, desglosados en componentes e indicadores; siendo uno de los componentes del criterio de idoneidad epistémica tener en cuenta una muestra representativa de la complejidad del objeto que se quiere enseñar (Breda, Font y Pino-Fan, 2018; Breda, Pino-Fan y Font, 2017). 


\section{Complejidad del objeto y criterios de Idoneidad didáctica}

La noción de idoneidad didáctica propuesta por el Enfoque Ontosemiótico de la Cognición e Instrucción Matemáticos (Breda, Font y Pino-Fan, 2018; Breda, Font, Lima y Pereira, 2018; Breda, Lima y Font, 2015), es una respuesta parcial a la siguiente problemática: ¿Qué criterios se deben utilizar para diseñar una secuencia de tareas, que permitan evaluar y desarrollar la competencia matemática de los alumnos y qué cambios se deben realizar en su rediseño para mejorar el desarrollo de esta competencia? Los criterios de idoneidad pueden servir primero para guiar los procesos de enseñanza y aprendizaje de las matemáticas y, segundo, para valorar sus implementaciones. Los criterios de idoneidad son útiles en dos momentos de los procesos de instrucción. A priori, los criterios de idoneidad son principios que orientan cómo se deben hacer las cosas. A posteriori, los criterios sirven para valorar el proceso de instrucción efectivamente implementado. En el EOS se consideran los siguientes criterios de idoneidad didáctica (Font, Planas y Godino, 2010):

$\checkmark \quad$ Idoneidad Epistémica, para valorar si las matemáticas que están siendo enseñadas son "buenas matemáticas".

$\checkmark \quad$ Idoneidad Cognitiva, para valorar, antes de iniciar el proceso de instrucción, si lo que se quiere enseñar está a una distancia razonable de aquello que los alumnos saben, y después del proceso, si los aprendizajes adquiridos están cerca de aquello que se pretendía enseñar.

$\checkmark$ Idoneidad Interaccional, para valorar si las interacciones resuelven dudas y dificultades de los alumnos.

$\checkmark$ Idoneidad Mediacional, para valorar la adecuación de los recursos materiales y temporales utilizados en el proceso de instrucción.

$\checkmark \quad$ Idoneidad Emocional, para valorar la implicación (intereses, motivaciones, ...) de los alumnos durante el proceso de instrucción.

$\checkmark \quad$ Idoneidad Ecológica, para valorar la adecuación del proceso de instrucción al proyecto educativo del centro, las directrices curriculares, las condiciones del entorno social y profesional.

La operatividad de los criterios de idoneidad exige definir un conjunto de componentes e indicadores observables, que permitan valorar el grado de idoneidad de cada uno de los criterios. 
Por ejemplo, todos seguramente estaremos de acuerdo en que es necesario implementar unas "buenas" matemáticas, pero podemos entender cosas muy diferentes por ello. Para algunos criterios, los descriptores son relativamente fáciles de consensuar (por ejemplo, para el criterio de idoneidad de medios), para otros, como es el caso de la idoneidad epistémica es más difícil. En Breda y Lima (2016), Seckel (2016) y Breda, Pino-Fan y Font (2017), se aporta un sistema de indicadores que sirve de guía de análisis y valoración de la idoneidad didáctica, que está pensado para un proceso de instrucción en cualquier etapa educativa. A continuación, se reproducen los componentes e indicadores del criterio de idoneidad epistémica:

Cuadro 1: Componentes e indicadores de la Idoneidad Epistémica

\begin{tabular}{|c|c|}
\hline Componentes & Indicadores \\
\hline Errores & $\begin{array}{l}\text { No se observan prácticas que se consideren incorrectas desde el } \\
\text { punto de vista matemático }\end{array}$ \\
\hline Ambigüedades & $\begin{array}{l}\text { No se observan ambigüedades que puedan llevar a la confusión a } \\
\text { los alumnos: definiciones y procedimientos clara y correctamente } \\
\text { enunciados, adaptados al nivel educativo al que se dirigen; } \\
\text { adecuación de las explicaciones, comprobaciones, demostraciones } \\
\text { al nivel educativo a que se dirigen, uso controlado de metáforas, } \\
\text { etc. }\end{array}$ \\
\hline Riqueza de procesos & $\begin{array}{l}\text { La secuencia de tareas contempla la realización de procesos } \\
\text { relevantes en la actividad matemática (modelización, } \\
\text { argumentación, resolución de problemas, conexiones, etc.). }\end{array}$ \\
\hline $\begin{array}{l}\text { Representatividad de la } \\
\text { complejidad del objeto } \\
\text { matemático }\end{array}$ & $\begin{array}{l}\text { Los significados parciales definiciones, propiedades, } \\
\text { procedimientos, etc.) son una muestra representativa de la } \\
\text { complejidad de la noción matemática que se quiere enseñar } \\
\text { contemplada en el currículo. } \\
\text { Los significados parciales (definiciones, propiedades, } \\
\text { procedimientos, etc.) son una muestra representativa de la } \\
\text { complejidad de la noción matemática que se quiere enseñar. } \\
\text { Para uno o varios significados parciales, muestra representativa de } \\
\text { problemas. } \\
\text { Para uno o varios significados parciales, uso de diferentes modos } \\
\text { de expresión (verbal, gráfico, simbólico...), tratamientos y } \\
\text { conversiones entre los mismos }\end{array}$ \\
\hline
\end{tabular}

Fuente: Breda y Lima (2016, p. 80). 
Tanto los componentes como los indicadores de los criterios de idoneidad didáctica se han confeccionado teniendo en cuenta las tendencias, los principios y los resultados de la investigación en el área de Didáctica de las Matemáticas. En particular, para la idoneidad epistémica se ha tenido en cuenta un principio fundamental del EOS que, con los matices propios de cada enfoque, es (o puede ser) asumido por otros enfoques teóricos del área. Nos referimos al principio que se puede formular de la siguiente manera: los objetos matemáticos emergen de las prácticas, lo cual conlleva su complejidad (Font, Godino y Gallardo, 2013; Rondero y Font, 2015). De este principio se deriva un componente (representatividad de la complejidad del objeto matemático) cuyo objetivo es que se tenga en cuenta, dentro de lo posible, dicha complejidad en el diseño y rediseño de las secuencias didácticas.

Este componente tiene su origen en la manera pragmatista de cómo se entiende el significado de un objeto matemático en el EOS y de cómo se entiende la emergencia de los objetos matemáticos a partir de las prácticas (explicado en la sección tres de este artículo). Desde un punto de vista pragmatista, el significado de un objeto matemático se entiende como el conjunto de prácticas en la que dicho objeto interviene de una manera determinante. Es decir, supone disponer de prácticas con respecto al campo de experiencia que el objeto abarca. Cuando se define el significado de un objeto matemático en términos de prácticas, tal como se propone en el pragmatismo, resulta que el significado de un objeto matemático queda ligado a otros significados y a otros objetos, puesto que en las prácticas interviene dicho objeto conjuntamente con otros objetos matemáticos. Este hecho, permite distinguir dos términos que resultan difíciles de diferenciar, nos referimos a los términos sentido y significado. En efecto, puesto que el objeto se puede relacionar con unos u otros objetos según el contexto, el tipo de notación, etc., para dar lugar a diferentes prácticas, podemos entender el sentido como un significado parcial, esto es, como un subconjunto (sentido) del sistema de prácticas en las que el objeto es determinante (significado).

El significado de un objeto matemático entendido como sistema de prácticas se puede parcelar en diferentes clases de prácticas más específicas que son utilizadas en un determinado contexto y con un determinado tipo de notación produciendo un determinado sentido. Cada contexto ayuda a generar sentido (permite generar un subconjunto de prácticas), pero no genera todos los sentidos. 
Un objeto matemático, que se ha originado como un emergente del sistema de prácticas que permite resolver un determinado campo de problemas, con el paso del tiempo queda enmarcado en diferentes programas de investigación. Cada nuevo programa de investigación permite resolver nuevos tipos de problemas, aplicar nuevos procedimientos, relacionar el objeto (y por tanto definir) de una manera diferente, utilizar nuevas representaciones, etc. De esta manera, con el paso del tiempo aparecen nuevos subconjuntos de prácticas (sentidos) que amplían el significado del objeto.

En el EOS (Font, Godino y Gallardo, 2013) se considera que, para analizar la actividad matemática, sea profesional o escolar, es necesario contemplar como mínimo los siguientes elementos: 1) notaciones, representaciones (lenguaje), 2) situaciones-problema 3) definiciones, 4) procedimientos, técnicas, ..., 5) proposiciones, propiedades, teoremas, etc., y 6) argumentos. Estos seis tipos de objetos primarios se articulan formando configuraciones de objetos llamadas epistémicas, y se pueden entender como un contexto intra matemático. Se trata de una herramienta que puede ser útil para describir la complejidad de los objetos matemáticos y de las prácticas de las cuales emergen. Por otra parte, cada una de estas configuraciones permite resolver un determinado tipo de problemas.

Desde esta perspectiva, un criterio de idoneidad de una trayectoria didáctica de enseñanza y aprendizaje para un objeto matemático es que el conjunto de prácticas implementadas sea un conjunto lo más representativo posible del sistema de prácticas que son el significado del objeto. Dicho en términos de contextos, hay que presentar a los alumnos una muestra de contextos intramatemáticos representativa, una muestra de contextos que permita construir una muestra representativa bien conectada de los diferentes sentidos del objeto. Por otra parte, una vez seleccionada una muestra representativa de contextos intramatemáticos, hay que seleccionar los contextos extra matemáticos que permiten hacer emerger las configuraciones epistémicas en las que se concretan dichos contextos intramatemáticos. Es decir, si se quiere conseguir que el alumno sea competente en la resolución de una variedad de problemas donde el objeto matemático en cuestión tiene un rol determinante, es necesario que el alumno disponga de una red de significados parciales bien conectados entre sí.

\section{La reflexión sobre la complejidad del objeto matemático en la formación de profesores}

El componente representatividad de la complejidad del objeto matemático que se quiere enseñar es uno de los componentes del criterio de idoneidad epistémica, que es una de las 
herramientas que se enseñan en diversos dispositivos formativos para desarrollar en los profesores la subcompetencia de análisis de la idoneidad didáctica de un proceso de instrucción, que a su vez es una de las subcompetencias que participan en la competencia de análisis e intervención didáctica, que es una de las competencias clave del modelo de Conocimientos y Competencias Didáctico-Matemáticas (CCDM), el cual ha sido desarrollado en diversos trabajos $^{1}$ (Breda, Pino-Fan y Font, 2017; Godino, Giacomone, Batanero y Font, 2017; Pino-Fan, Assis \& Castro, 2015; Pino-Fan, Font y Breda, 2017), y que está basado en el Enfoque ontosemiótico del Conocimiento y la Instrucción Matemáticos (Godino, Batanero y Font, 2007; 2019). La subcompetencia de análisis de la idoneidad didáctica, resulta clave para la reflexión global del docente sobre su práctica, en particular para su valoración y mejora progresiva.

En diferentes procesos de formación de profesores de matemáticas de España, México, Brasil, Ecuador, Chile, Panamá, Costa Rica, Venezuela y Argentina (Breda, 2016; Breda, Font y Lima, 2015; Breda y Lima, 2016; Breda, Pino-Fan y Font, 2016 y 2017; Esqué y Breda, 2021; Ferreres y Vanegas, 2015; Giménez, Vanegas, Font y Ferreres, 2012; Giménez, Font y Vanegas, 2013; Hummes, Font y Breda, 2019; Hummes, Font, Breda y Seckel, en prensa; Pino-Fan, Godino y Font, 2018; Morales-Maure, 2019; Morales-Maure, Durán-González y GarcíaVázquez, 2019; Morales-Maure, Durán-González, Pérez-Maya y Bustamante, 2019; MoralesLópez y Font, 2017 y 2019; Pochulu, Font y Rodríguez, 2016; Ramos, 2006; Ramos y Font, 2008; Seckel, 2016; Seckel y Font, 2020), se han realizado un conjunto de investigaciones que tienen como finalidad investigar el uso del constructo criterios de idoneidad didáctica, propuestos por el EOS, como herramienta para organizar la reflexión del profesor sobre su práctica, cuando esta reflexión está orientada hacia la valoración y mejora de la práctica, con el objetivo de desarrollar en los profesores la subcompetencia de análisis de la idoneidad didáctica de un proceso de instrucción.

Estas investigaciones han puesto de manifiesto los siguientes aspectos: 1) aunque no se incorpore explícitamente la enseñanza de los componentes e indicadores de los criterios de idoneidad didáctica, algunos de ellos, y en particular el componente <<muestra representativa de la complejidad del objeto matemático>>, están presentes de manera implícita cuando los profesores o futuros profesores hacen valoraciones de propuestas didácticas (suyas o de otros)

\footnotetext{
${ }^{1}$ Las publicaciones realizadas en el marco del EOS, aplicando el modelo CCDM, están disponibles en el sitio web http://enfoqueontosemiotico.ugr.es (entrada sobre Formación de profesores).
} 
(Breda, 2016; Breda y Lima, 2016; Breda, Pino-Fan y Font, 2017). 2) Incorporar el componente <<muestra representativa de la complejidad del objeto matemático〉> para valorar la idoneidad epistémica de un proceso de enseñanza y aprendizaje, no es tarea fácil, ni para los formadores ni para sus alumnos (futuros profesores o profesores en servicio), pero se puede enseñar como parte del proceso de formación del profesorado (Font, Breda y Pino-Fan, 2017; Calle, Breda y Font, en prensa; Calle y Breda, 2019; Seckel y Font, 2020). En estos dispositivos formativos, se hace hincapié en la necesidad de realizar un estudio preliminar orientado a la reconstrucción de un significado global sobre el objeto matemático que se quiere enseñar para poder ser conscientes de su complejidad.

Los autores de este artículo, conjuntamente con otros investigadores, están desarrollando el diseño y la implementación de recursos formativos que promuevan la realización de este tipo de análisis valorativo por parte de los profesores (en formación o en servicio) utilizando la herramienta criterios de idoneidad didáctica. A continuación, ponemos un ejemplo para ilustrar algunos momentos del proceso de enseñanza y aprendizaje del componente representatividad de la complejidad del objeto matemático realizado en un dispositivo de formación inicial de profesores de matemáticas, implementado en España (Font, Breda y Pino-Fan, 2017). Se trata de un ejemplo que presenta el uso de dicho componente en el proceso de reflexión, explicado en el Trabajo de Fin de Máster (TFM) de una futura profesora, con el fin de mejorar la implementación de una unidad didáctica (UD) diseñada e implementada anteriormente. El uso de los criterios de idoneidad didáctica fue enseñado en el proceso formativo de esta futura profesora como una herramienta metodológica para promover y apoyar la reflexión sobre su práctica.

La futura profesora, en su TFM, presenta la valoración y el rediseño de una propuesta didáctica sobre la función de segundo grado, para un grupo de alumnos del cuarto año de la Enseñanza Secundaria Obligatoria (ESO) de España (15-16 años de edad). En el tercer capítulo, donde realiza la valoración de los seis criterios de idoneidad didáctica, cuando valora la idoneidad epistémica, con relación al componente <<muestra representativa de la complejidad del objeto matemático > dice que éste se debe mejorar en el rediseño de la unidad didáctica de cara a futuras implementaciones y argumenta su valoración negativa de dicho componente de la manera siguiente: 
El principal significado parcial que se ha trabajado en la UD ha sido el de la representación de la función explicita, por lo que no podemos considerarlos como una muestra representativa de la complejidad de la función cuadrática. Han faltado significados parciales y sus conexiones.

La muestra de actividades presentadas en la UD no ha sido representativa. Han faltado problemas de contextos significativos de la aplicación de la parábola, como pueden ser de tiro parabólico, de fuentes (por ejemplo, Montjuïc), de luz de faros, etc. (Font, Breda y Pino-Fan, 2017, p. 251).

Es importante resaltar que, en los diferentes dispositivos de formación, la necesidad de contemplar la complejidad del objeto matemático que se quiere enseñar se argumenta de varias formas, siendo una de las más importantes el hecho de que, en muchos currículos, se pretende que las matemáticas enseñadas se apliquen a la resolución de problemas (en especial, problemas de contexto extra matemático). Se les hace observar a los participantes que los problemas donde el uso de un determinado objeto matemático es determinante, son de diversos tipos y que, para su resolución, se ponen en funcionamiento diferentes significados parciales del objeto que se pretende enseñar. Por tanto, o bien se enseña una muestra representativa de significados parciales del significado global del objeto matemático en cuestión, o bien sólo los alumnos más creativos pueden aplicar el objeto a los diversos problemas.

\section{Agradecimientos}

Trabajo desarrollado en el marco del proyecto de investigación en formación de profesorado: PGC2018-098603-B-I00 (MCIU/AEI/FEDER, UE).

\section{Referencias}

Arcavi, A. (2000). Problem-driven research in mathematics education. Journal of Mathematical Behavior, 19, 141-173.

Balcaza, T., Contreras, A. y Font, V. (2017). Análisis de libros de texto sobre la optimización en el bachillerato. Bolema, 31(59), 1061-1081.

Breda, A. (2016). Melhorias no ensino de matemática na concepção de professores que realizam o mestrado PROFMAT no Rio Grande do Sul: uma análise dos trabalhos de conclusão de curso (Tesis doctoral no publicada). Pontifícia Universidade Católica do Rio Grande do Sul.

Breda, A., Font, V., y Lima, V. M. R. (2015). A noção de idoneidade didática e seu uso na formação de professores de matemática. Jornal Internacional de Estudos em Educação Matemática, 8(2), 1-41. doi: 10.17921/2176-5634.2015v8n2p\%25p 
Breda, A., Font, V., Lima, V. M. R., Pereira, M. V. (2018). Componentes e indicadores de los criterios de idoneidad didáctica desde la perspectiva del enfoque ontosemiótico. Transformación, 14(2), 162-176.

Breda, A., Font, V., y Pino-Fan, L. (2018). Criterios valorativos y normativos en la Didáctica de las Matemáticas: el caso del constructo idoneidad didáctica. Bolema, 32(60), 255 - 278.

Breda, A., Lima, V. M. R. (2016). Estudio de caso sobre el anális didáctico realizado en un trabajo final de un máster para profesores de matemáticas en servicio. REDIMAT - Journal of Research in Mathematics Education, 5(1), 74-103. doi: 10.4471/redimat.2016.1955

Breda, A., Pino-Fan, L., y Font, V. (2016). Establishing criteria for teachers' reflection on their own practices. In Csíkos, C., rausch, A., \& Szitányi, J. (Eds.), Proceedings of the 40th Conference of the International Group for the Psychology of Mathematics Education (Vol. 1, pp. 283). Szeged, Hungary: PME.

Breda, A., Pino-Fan, L., y Font, V. (2017). Meta didactic-mathematical knowledge of teachers: criteria for the reflection and assessment on teaching practice. Eurasia Journal of Mathematics, Science and Technology Education, 13(6), 1893-1918. doi: 10.12973/eurasia.2017.01207a.

Calle, E., Breda, A. (2019). Reflexión sobre la complejidad de los objetos matemáticos en la formación inicial de profesores. En Daniel Aguilar, Martha Cobos, Luis Claudio Cortés, Enma Campozano (Eds), La Investigación Educativa en un Mundo en Constante Transformación (pp. 29-50). Cuenca: ASEFIE.

Calle, E., Breda, A. y Font, V. (en prensa). ¿Qué significado atribuyen a la media aritmética profesores de matemáticas en ejercicio? Revista Acta Latinoamericana de Matemática Educativa.

Contreras, A., García, M., y Font, V. (2012). Análisis de un proceso de estudio sobre la enseñanza del límite de una función. Bolema, 26(42B), 667-690.

Duval, R. (2002). Representation, vision and visualization: cognitive functions in mathematical thinking. Basic issues for learning. In F. Hitt, (Ed.), Representations and Mathematics Visualization (pp. 311-335). North American Chapter of PME: Cinvestav-IPN, México.

Esqué, D., Breda, A. (2021). Valoración y rediseño de una unidad sobre proporcionalidad, utilizando la herramienta Idoneidad Didáctica. Uniciencia, 35(1), 1-19.

Ferreres, S., y Vanegas, Y. (2015). Uso de criterios de calidad en la reflexión sobre la práctica de los futuros profesores de secundaria de matemáticas. Procedia, 196, 219-225.

Font, V., Breda, A., y Pino-Fan, L. (2017). Análisis didáctico en un trabajo de fin de máster de un futuro profesor. En J. M. Muñoz-Escolano, A. Arnal-Bailera, P. Beltrán-Pellicer, M. L. Callejo y J. Carrillo (Eds.), Investigación en Educación Matemática XXI (pp. 247-256). Zaragoza: SEIEM.

Font, V., Breda, A., y Seckel, M. J. (2017). Algunas implicaciones didácticas derivadas de la complejidad de los objetos matemáticos cuando estos se aplican a distintos contextos. Revista Brasileira de Ensino de Ciência e Tecnologia, 10(2), 1-23.

Font, V., Godino, J. D., y D'Amore, B. (2007). An onto-semiotic approach to representations in mathematics education. For the Learning of Mathematics, 27(2), 1-7. 
Font, V., Godino, J. D., y D’Amore, B. (2010). Representations in matematics education. An onto-semiotic approach. Jornal Internacional de Estudos em Educação Matemática, 2, 5886.

Font, V., Godino, J. D., y Gallardo, J. (2013). The emergence of objects from mathematical practices. Educational Studies in Mathematics, 82, 97-124.

Font, V., Godino, J. D., Planas, N., y Acevedo, J. I. (2010). The object metaphor and sinecdoque in mathematics classroom discourse. For the Learning of Mathematics, 30, 15-19.

Font, V., Planas, N., y Godino, J. D. (2010). Modelo para el análisis didáctico en educación matemática. Infancia y Aprendizaje, 33(1), 89-105.

Font, V., y Peraire, R. (2001) Objetos, prácticas y ostensivos asociados. el caso de la cisoide, Educación matemática, 13(2), 55-67.

Giménez, J., Font, V., y Vanegas, Y. (2013). Designing professional tasks for didactical analysis as a research process. In Margolinas, C. (Ed.), Task Design in Mathematics Education. Proceedings of ICMI Study 22: Oxford, 2013.

Giménez, J., Vanegas, Y., Font, V., y Ferreres, S. (2012). El papel del trabajo final de máster en la formación del profesorado de matemáticas. UNO. Revista de Didáctica de las Matemáticas, 61, 76-86.

Godino, J. D., Batanero, C., y Font, V. (2007). The onto-semiotic approach to research in mathematics education. ZDM. The International Journal on Mathematics Education, 39(1), 127-135.

Godino, J. D., Batanero, C., y Font, V. (2019). The Onto-semiotic Approach: implications for the prescriptive character of didactics. For the Learning of Mathematics, 39(1), 37-42.

Godino, J. D., Bencomo, D., Font, V. y Wilhemi, M. R. (2006). Análisis y Valoración de la Idoneidad Didáctica de Procesos de Estudio de las Matemáticas. Paradigma, 27(2), 221252.

Godino, J. D., y Font, V. (2010). The theory of representations as viewed from the onto-semiotic approach to mathematics education. Mediterranean Journal for Research in Mathematics Education, 9(1), 189-210.

Godino, J. D., Font, V., Wilhelmi, M. R., y Arrieche, M. (2009). ¿Alguien sabe qué es el número?. Unión, 19, 34-46.

Godino, J. D., Giacomone, B., Batanero, C., y Font, V. (2017). Enfoque ontosemiótico de los conocimientos y competencias del profesor de matemáticas. Bolema, 31(57), 90-113.

Gordillo, W., y Pino-Fan, L. (2016). Una propuesta de reconstrucción del significado holístico de la antiderivada. BOLEMA, 30(55), 535-558.

Gordillo, W., Pino-Fan, L., Font, V. y Ponce-Campuzano, J. (2018). Algunas tareas para evaluar la comprensión sobre el objeto matemático antiderivada. Academia y virtualidad, 11(2), 117.

Hamilton, M. L., Smith, L. \& Worthington, K. (2008) Fitting the Methodology with the Research: An exploration of narrative, self-study and auto-ethnography, Studying Teacher Education, 4(1), 17-28, DOI: 10.1080/17425960801976321 
Hitt, F. (1998). Difficulties in the articulation of different representations linked to the concept of function. Journal of Mathematical Behavior, 17(1), 123-134.

Hummes, V. B., Font, V., Breda, A., (2019). Combined Use of the Lesson Study and the Criteria of Didactical Suitability for the Development of the Reflection on the own Practice in the Training of Mathematics Teachers, Acta Scientiae, 21(1), 64-82.

Hummes, V. B., Font, V., Breda, A. y Seckel, M. J. (en prensa). Criterios de idoneidad didáctica en una clase basada en el Lesson Study. Praxis \& Saber.

Monje, Y., Seckel, M.J. y Breda, A. (2018). Tratamiento de la Inecuación en el Currículum y Textos Escolares Chilenos. Bolema, 32(61), 480-502.

Morales-López, Y., y Font, V. (2017). Análisis de la reflexión presente en las crónicas de estudiantes en formación inicial en educación matemática durante su periodo de práctica profesional. Acta Scientiae, 19(1), 122-137.

Morales-López, Y., y Font, V. (2019). Evaluation by a teacher of the suitability of her mathematics class. Educaçao e Pesquisa, 45, e189468.

Morales-Maure, L. (2019). Competencia de análisis e intervención didáctica del docente de primaria en Panamá. Tesis doctoral no publicada). Universidad de Barcelona, España.

Morales-Maure, L., Durán-González, R., Pérez-Maya, C., \& Bustamante, M. (2019). Hallazgos en la formación de profesores para la enseñanza de la matemática desde la idoneidad didáctica. Experiencia en cinco regiones educativas de Panamá. Inclusiones, 6(2), 142-162.

Morales-Maure, L., Durán-González, R., \& García-Vázquez, E. (2019). Intervención formativa para el aprendizaje de las matemáticas: una aproximación desde un diplomado. Conrado, 15(69), 7-19.

Niss, M. (2018). The very multi-faceted nature of mathematics education research. In E. Bergqvist, M. Österholm, C. Granberg, \& L. Sumpter (Eds.), Proceedings of the 42th Conference of the International Group for the Psychology of Mathematics Education, (Vol. 1, pp. 35-50). Umeå, Sweden: PME.

Pino-Fan, L., Assis, A., \& Castro, W. F. (2015). Towards a methodology for the characterization of teachers' didactic-mathematical knowledge. Eurasia Journal of Mathematics, Science \& Technology Education, 11(6), 1429-1456. doi: 10.12973/eurasia.2015.1403a.

Pino-Fan, L., Castro, W. F., Godino, J. D., y Font, V. (2013). Idoneidad epistémica del significado de la derivada en el currículo de bachillerato. Paradigma, 34(2), 123 - 150.

Pino-Fan, L., Font, V., y Breda, A. (2017). Mathematics teachers' knowledge and competences model based on the onto-semiotic approach. In B. Kaur, W. K. Ho, T. L. Toh \& B. H. Choy (Eds.), Proceedings of the 41st Conference of the International Group for the Psychology of Mathematics Education (Vol. 4, pp. 33-40). Singapure: PME.

Pino-Fan, L., Font, V., Gordillo, W., Larios, V., y Breda (2018). Analysis of the meanings of the antiderivative used by students of the first engineering courses. International Journal of Science and Mathematics Education. 16(6), 1091-1113. doi: 10.1007/s10763-01.

Pino-Fan, L., Godino, J. D., y Font, V. (2018). Assessing key epistemic features of didacticmathematical knowledge of prospective teachers: the case of the derivative. Journal of Mathematics Teacher Education, 21(1), 63-94. 
Pino-Fan, L., Godino, J. D. y Font, V. (2011). Faceta epistémica del conocimiento didácticomatemático sobre la derivada. Educação Matemática Pesquisa, 13(1), 141-178.

Pochulu, M., Font, V., Rodríguez, M. (2016). Desarrollo de la competencia en análisis didáctico de formadores de futuros profesores de matemática a través del diseño de tareas. Revista Latinoamericana de Investigación en Matemática Educativa-RELIME, 19(1), 71-98.

Ramos, A. B. (2006). Objetos personales, matemáticos y didácticos, del profesorado y cambios institucionales. el caso de la contextualización de las funciones en una facultad de ciencias económicas y sociales (Tesis doctoral no publicada). Universitat de Barcelona, España.

Ramos, A. B., y Font, V. (2008). Criterios de idoneidad y valoración de cambios en el proceso de instrucción matemàtica. Revista Latinoamericana de Investigación en Matemática Educativa-RELIME, 11(2), 233-265.

Rondero, C., y Font, V. (2015). Articulación de la complejidad matemática de la media aritmética. Enseñanza de las Ciencias, 33(2), 29-49.

Seckel, M. J. (2016). Competencia en análisis didáctico en la formación inicial de profesores de educación general básica con mención en matemática (Tesis doctoral no publicada). Universidad de Barcelona, España.

Seckel, M., \& Font, V. (2020). Competencia reflexiva en formadores del profesorado en matemáticas. Magis, 12(25), 127-144.

Wittgenstein, L. (1978). Remarks on the foundations of mathematics (3rd. ed.). Oxford, England: Basil Blackwell. 